\title{
DNA-Lipid Hybrid Films Derived from Chiral Lipids
}

\author{
By Naoya OGATA ${ }^{1, *}$ and Kanji YAMAOKA ${ }^{2}$
}

Chiral lipids (quarternized form) were synthesized from L-,D.-, racemic alanine, L-phenyl alanine and L-glutamic acid having $\mathrm{C} 10$ alcohol unit as an ester group and DNA hybrid complexes were prepared from these chiral lipids. The as-cast DNA-L-alanineC10 complex film showed a self-assembled structure which was enhanced by water, while no self assembled structure was observed for the DNA-D-or racemic alanine C10 complex film.

DNA-L-Glutamic acidC10 (GluC10) lipid and L-phenylalanineC10 (PhAlC10) lipid complexes did not form a selfassembled structure which was clearly observed for the DNA-L-alanineC10 complex. Two C10 alkyl groups of GluC10 and bulky benzyl group of PhAlC10 surrounding DNA double helix may disturb the formation of the self-assembled structure. KEY WORDS: DNA / Chiral Lipids / L-Alanine / L-Glutamic acid / L-Phenylalanine / DNA-Lipid Complex /

Deoxyribonucleic acid (DNA) which contains gene information of all living things is a water-soluble polymer with a very high molecular weight of over billions. DNA molecules are an anionic polyphosphate with sodium cation as a counter ion (DNA-Na), which can be replaced by lipids having quarternized ammonium cation to form DNA-lipid hybrid complexes. The ion exchange reaction of sodium cation of DNA takes place by simply mixing an aqueous solution of DNA-Na with an aqueous solution of excess amount (more than twice molar ratios) of lipids at ambient temperature and a DNA-lipid hybrid complex is precipitated out of the aqueous solution. The DNA-lipid hybrid complexes become insoluble in water and become soluble in polar organic solvents such as alcohols, and transparent films of DNA-lipid hybrid complex are obtained by a simple solvent casting method. ${ }^{1}$ Thus, various applications of DNA have been developing in wide areas of photonic or electronic devices. $^{2-6}$

Physical properties of the DNA-lipid complexes are strongly dependent on kinds of lipid in terms of number of alkyl chain length of lipids. ${ }^{7}$ It was reported ${ }^{8}$ that when a cationic lipid having chiral structure such as cholesteryl $3 \beta-N$-((dimethylamino)ethy)carbamate (DC-Chol) was used to form a binary complex of DNA and cationic lipid, a self-assembled structure of DNA-DC-Chol complex was obtained. It is expected that more simple chiral lipids may induce to construct a strong selfassembled structure of DNA hybrid film. This paper describes characteristic features of DNA-chiral lipid complex films which were derived from amino acids.

\section{EXPERIMENTAL}

\section{Preparation of Chiral Lipids from Alanine, Phenyl Alanine and Glutamic Acid}

Chiral lipids with alkyl group of $\mathrm{C}=10$, n-decanol as an ester group of L-alanine (abbrebiated as L-alanineC10) were synthesized and purchased from the Konan Kako Co., by following synthetic route as shown in Scheme 1 where yields of each synthetic steps were also shown. Precise reaction conditions were not opened by the company. L-,D-, and racemic lipids were synthesized by the same synthetic route. When long alkyl alcohols of more than $\mathrm{C}=16$ were used to prepare alanine lipids, lipids were not soluble in water so that alcohol of $\mathrm{C}=10$ was used.

Chiral lipid having two $\mathrm{C} 10$ ester chains was prepared from L-glutamic acid and L-phenyl alanine by the similar method as alanine-derived lipid, which is shown in Scheme 2.

\section{Preparation of DNA-Chiral Lipid Complex and Films}

Pure DNA derived from Salmon was dissolved an amount of $1 \mathrm{~g}$ in $100 \mathrm{~mL}$ water. Chiral lipids derived from L-, D- or racemic alanine were dissolved in a twice molar amount of each $2 \mathrm{~g}$ in $100 \mathrm{~mL}$ of water. The DNA-alanine-derived lipid complexes were obtained by adding dropwisely the aqueous solution of these alanine-derived lipids into the aqueous solution of DNA, followed by filtering and drying in vacuum at ambient temperature. Films of the DNA-alanine-derived lipid complexes were obtained by casting solutions of the DNA-lipid complexes in hexafluroisopropanol $(1 \mathrm{~g} / 100 \mathrm{~mL})$ onto a Teflon-coated plate, followed by drying in vacuum to obtain transparent films. The DNA-lipid complexes were soluble in ethanol right after the preparation of the complexes, while they became insoluble in any other organic solvents except hexafluroroisopropanol, by standing possibly owing to recrystallization.

The chiral lipids derived from L-glutamic acid (L-GluC10) and L-phenylalanine (L-PhAlC10) were insoluble in water, so the preparation of the DNA-lipid complex was performed by following method: $1 \mathrm{~g}$ of DNA dissolved in $100 \mathrm{~mL}$ of water, followed by adding dropwisely $100 \mathrm{~mL}$ of ethanol solution of $1 \mathrm{~g}$ of L-GluC10 or L-PhAlC10, and the DNA-L-GluC10 complex was precipitated, followed by filtration and washing with water and drying.

Structure of these DNA-chiral lipid complexes were confirmed by IR and NMR analyses.

\footnotetext{
${ }^{1}$ Ogata Research Laboratory, Ltd., 1-3-1 Kashiwadai-minami, Chitose 066-0009, Japan

${ }^{2}$ NEDO Fellow, Ogata Research Laboratory, Ltd., 1-3-1 Kashiwadai-minami, Chitose 066-0009, Japan

*To whom correspondence should be addressed (E-mail: n-ogata@ photon.chitose.ac.jp).
} 


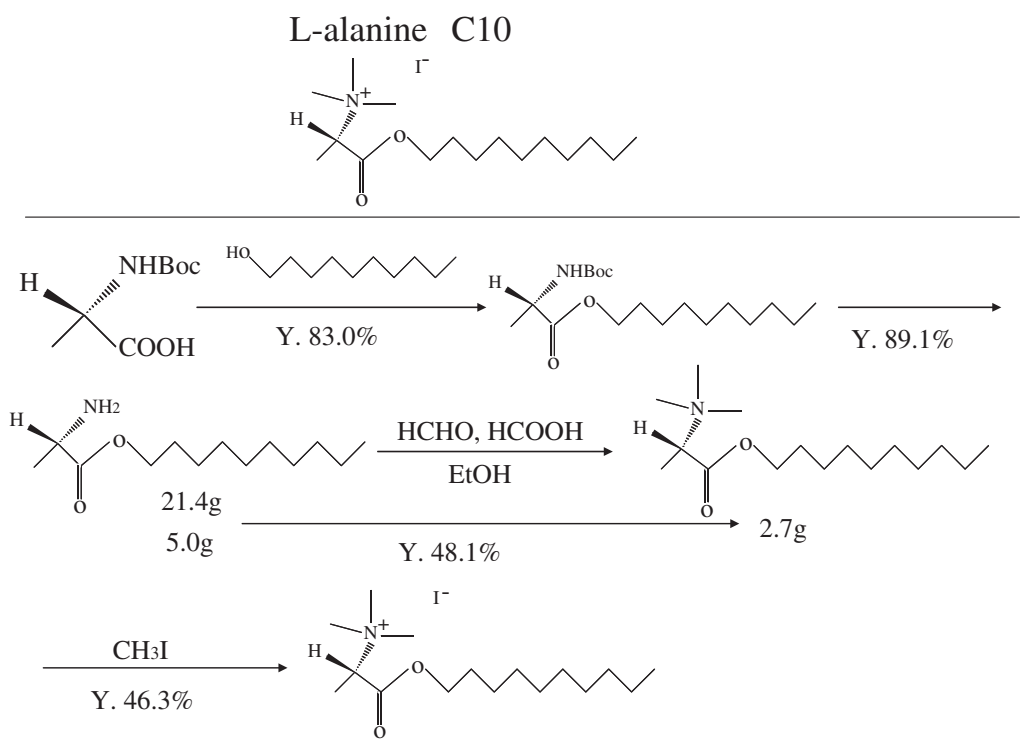

Scheme 1. Synthetic method of L-alanine C10.

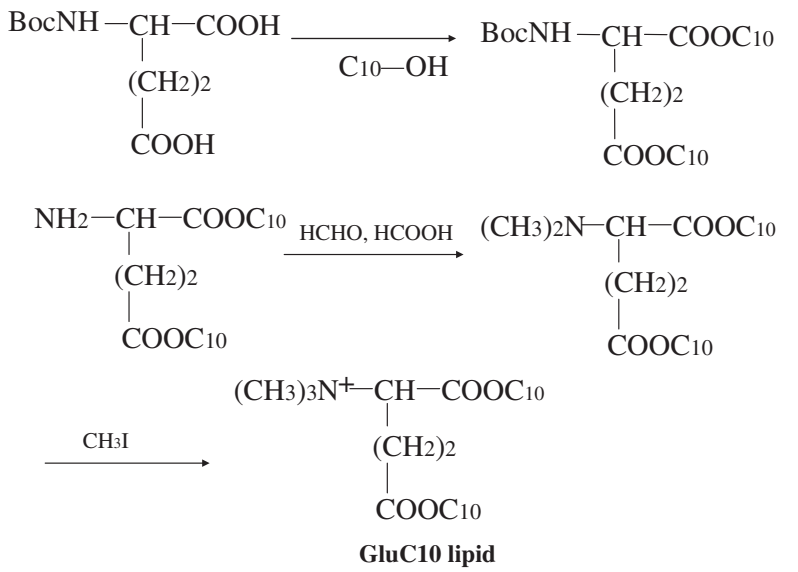

Scheme 2. Synthesis of GluC10 lipid.

\section{Physical Properties}

Tensile strength and elongation of films were measured by Toyo Tensiron instrument at ambient temperature after drying the films in vacuum. Water absorption behaviors of the films were determined by keeping the films under various relative humidity at ambient temperature for $2 \mathrm{~d}$, followed by measuring weight increases of the films. Microscopic pictures of the films were observed under a cross-polarized microscope, by rotating films. Surface structures of the films were observed by a JEOL scanning probe microscope.

$\mathrm{X}$-Ray refraction of various DNA-lipid membranes was measured by Rigaku-denki X-ray refractometer.

\section{RESULTS AND DISCUSSION}

DNA-Alanine-derived Lipid Complex Films

Figure 1 shows IR spectrum of DNA-L-alanine C10 film. A peak of an ester group of L-alanineC10 is confirmed at

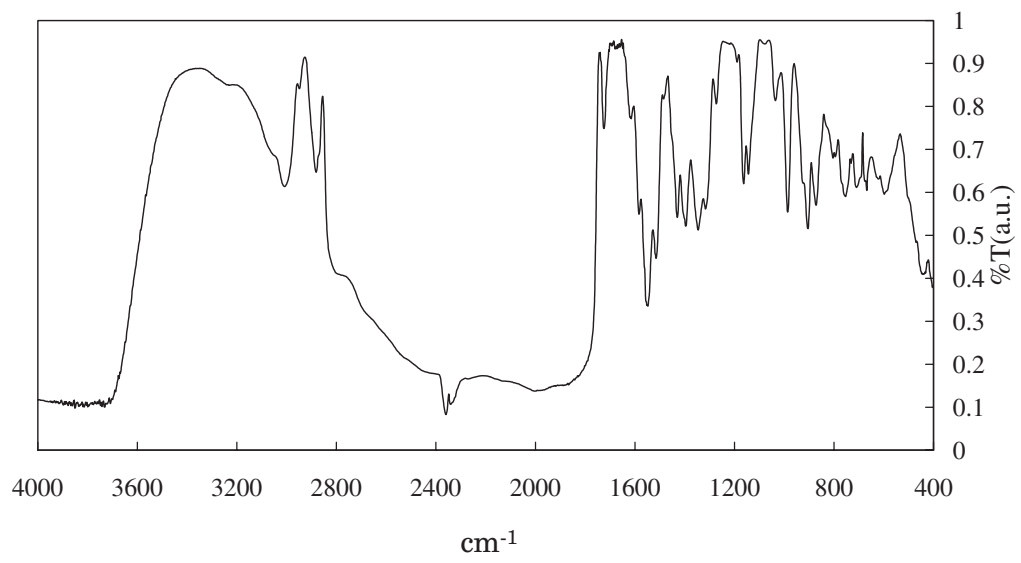

Figure 1. IR spectrum of DNA-Lalanine comlex film 


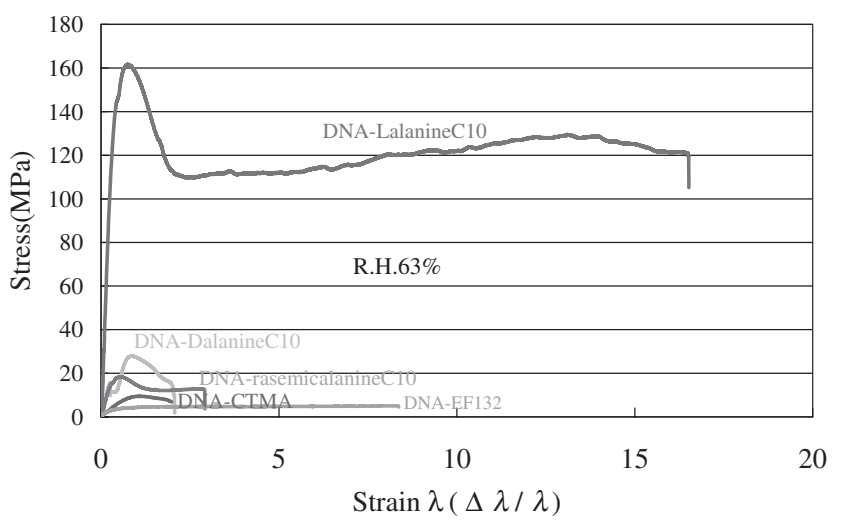

Figure 2. Stress-Strain Curves of various DNA-lipid complex films.

$1710 \mathrm{~cm}^{-1}$. The molar ratios of amphiphile to nucleotide of polyion complexes was identified as 1 to 1 by a phosphoric acid assay.

Figure 2 shows stress-strain curves of DNA-lipid films derived from DNA and alanineC10, in comparison with film properties of DNA-CTMA (cetyltrimethylammonium chloride) and DNA-EF132 (Asahi Glass Co. made, fluoroalkylammonium chloride, structure is unknown) complexes. It can be clearly pointed out in Figure 2 that the film derived from DNAL-alanineC10 indicated a characteristic feature of mechanical properties in terms of both strength and elongation, which were almost more than 10 times higher in strength and also in elongation than those of DNA-CTMA complex film. On the other hand, the films from DNA-D-alanineC10 and also DNAracemic alanineC10 showed almost similar mechanical properties as the DNA-CTMA complex film. The DNA-EF132 films indicated a longer breaking elongation of $25 \mathrm{~mm}$ because of slippery fluorinated alky chain than that of DNA-CTMA, DNA-D- or racemic alanineC10 films. However, the elongation of the DNA-L-alanineC10 film was twice as much as that of DNA-EF132 film. Normally, as mechanical strength of films increases, elongation decreases on the opposite direction because of oriented structure of polymers such as nylon or polyester fibers. No other polymers are known to show both enhanced mechanical strength and elongation except demon spider silk and the physical properties of the DNA-alanineC10 lipid are very unique.

Water absorption behaviors of various DNA-lipid films were summarized in Figure 3, where it is seen that DNA-LalanineC10 film showed much less water absorption than other DNA-lipid complex films, especially under high relative humidity of more than $80 \%$. DNA-lipid complexes containing one single alky chain of $\mathrm{C}=12,14$ and 16 showed a high water absorption at high relative humidity of more than $80 \%$, while water absorption values decreased with increasing carbon number of lipids because of hydrophobic increases owing to long alkyl chains. ${ }^{7}$ Water absorptions of DNA-DalanineC10 or DNA-racemic alanineC10 were less than those of DNA-single alkyl chain lipid of $\mathrm{C}=12$.

Figure 4 indicates cross-polarized microscopic pictures of DNA-L-alanineC10, DNA-D-alanineC10 and DNA-racemic alanineC10 films under $100 \%$ relative humidity at ambient temperature, respectively. When a polarizer was rotated at $45^{\circ}$ angle, no change of the film picture for the DNA-D-alanine C10 was observed, showing only a dark background. On the other hand, the DNA-L-alanineC10 film showed a clear bright refractive picture by rotating a polarizer at $45^{\circ}$ angle, indicating an oriented structure of DNA-L-alanineC10 molecules. DNA-racemic alanineC10 film showed a little bright picture by rotating a polarizer at $45^{\circ}$ angle. It is quite interesting that the DNA-D-alanineC10 which was derived from an another isomer of alanineC 10 did not show an oriented structure and DNA-racemic alanineC10 showed a little oriented structure under a cross-polarized microscope. As-cast film

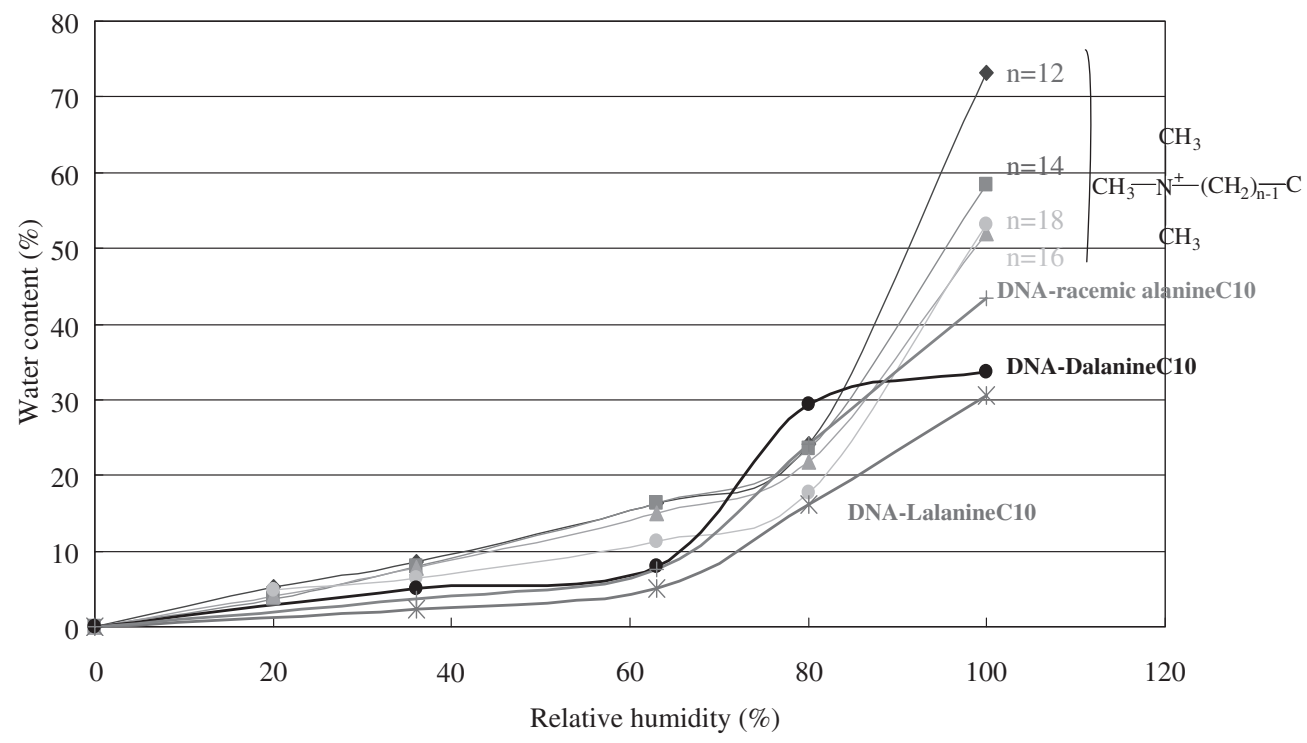

Figure 3. Water absorption behaviors of various DNA-lipid complex films. 


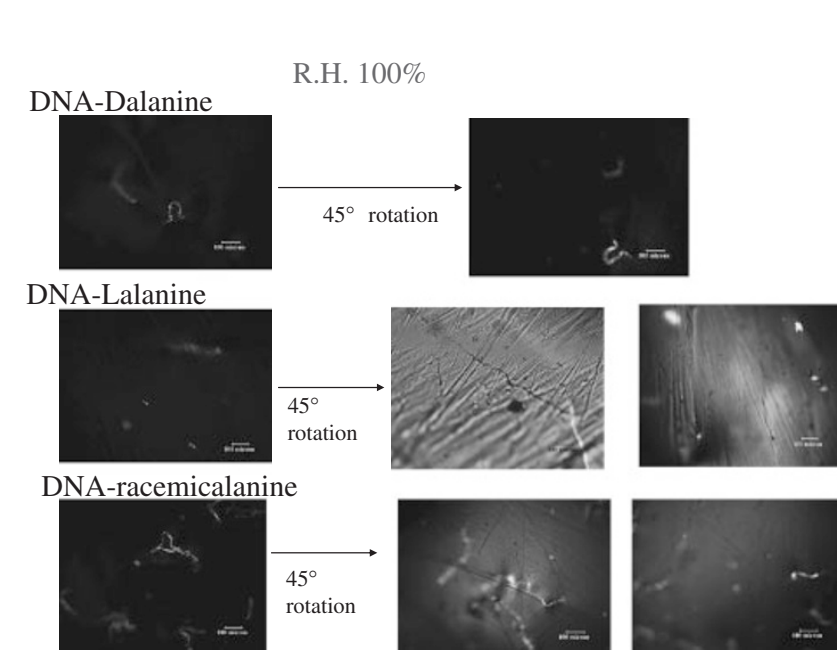

Figure 4. Cross-polarized pictures of as-cast DNA-alanine C10 films.

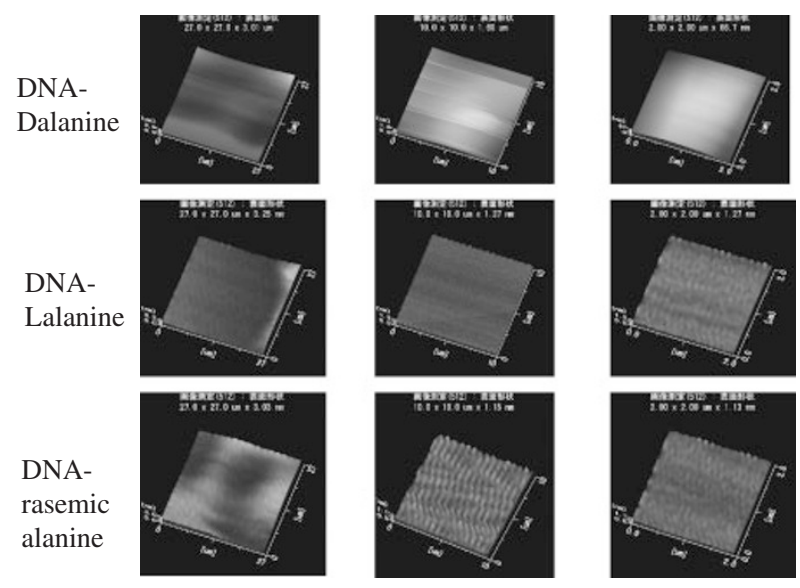

Figure 6. SPM pictures of surface structures of DNA-alanine C10 complex films.

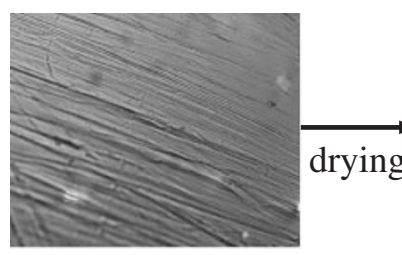

R.H. $100 \%$ $\left(45^{\circ}\right.$ rotation $)$

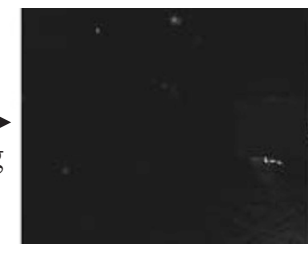

R.H.0\% ( $45^{\circ}$ rotation $)$

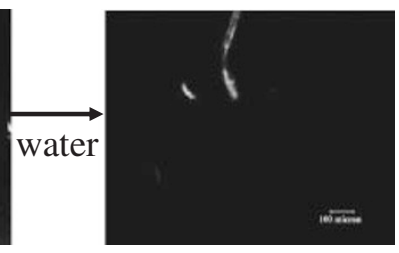

R.H.63\% ( $45^{\circ}$ rotation)

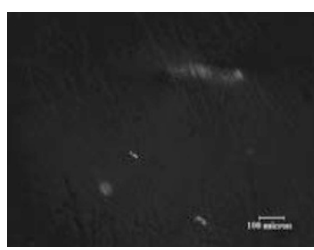

R.H. $80 \%$ ( $45^{\circ}$ rotation $)$

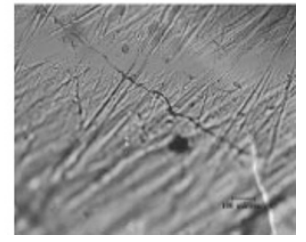

R.H. $100 \%$

$\left(45^{\circ}\right.$ rotation )

Figure 5. Effect of water for reorientation of DNA molecules.

of the DNA-L-alanineC10 showed a clear oriented structure as shown in Figure 5, while a dark picture was observed after drying the film at $0 \%$ relative humidity. However, gradual increases in relative humidity from $63 \%, 80 \%$ to $100 \%$ resulted in a recovery of an oriented structure of the film, as shown in Figure 5. In a dry state, dark field was confirmed even if turned 45 degrees. Drying process may cause a randomization of oriented structure of DNA-L-alanineC10 complex molecules by heating, while increasing water content in the film may stimulate the reconstruction of a self-assembled structure of the DNA-L-alanineC10 complex molecules, possibly by a plasticizing effect of water.

Surface structures of these as-cast DNA-alanineC10 complex films were observed by a scanning probe microscope (SPM) and SPM pictures are summarized in Figure 6 which showed each three different magnifying scales. The DNA-DalanineC10 film showed a smooth surface without any particular patterns, while the DNA-L-alanineC10 film exhibited an uneven surface with a regular interval pattern. The DNA-racemic alanineC10 film also showed the similar uneven surface with a regular interval pattern as the DNA-LalanineC10 film, as shown in Figure 7. These regular uneven pattern of these films may be ascribed to an oriented structure of the DNA-L-alanineC10 molecules. The lower water absorption behaviors of the DNA-L-alanineC10 complex film which are shown in Figure 3 can be explained by the oriented structure to increase crystallization which would decrease the water absorption.

It is not clear why the DNA-D-alanineC10 which is an aother side of optical isomers of the DNA-L-alanineC10, did not showed a self-assembled oriented structure. The DNAracemic alanineC 10 indicated a little self-assembled structure which may be ascribed to a half amount of L-alanine units. The enhanced self-assembled structure of the DNA-L-alanineC10 

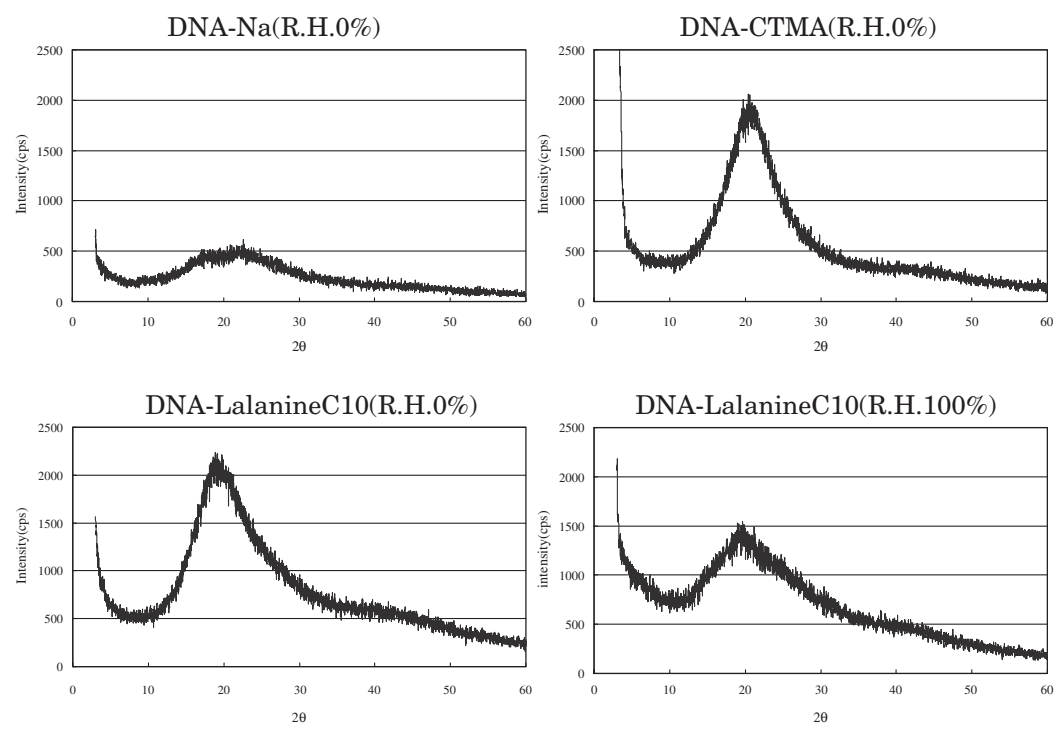

Figure 7. X-Ray refraction patterns of DNA-lipid complex films.

molecules may be related with left-hand side helical directions of DNA molecules as the L-alanine unit is attached to DNA molecules as side chains, and D-alanine unit may not fit with the helical directions of DNA molecules to organize a selfassembled structure.

In order to confirm the self-assembled structures of the DNA-lipid complex films, X-ray refraction of these films were measured and results are shown in Figure 7. DNA-Na film showed an amorphous refraction pattern, while DNA-CTMA film showed a crystalline pattern. It seems that a surface morphology of the SPM pictures may coincide with this result. The DNA-L-alanineC10 lipid complex film showed a little sharper refraction pattern with a shift of $2 \theta$ of 18 , toward lower value in comparison with the $2 \theta$ value of 20 of DNA-CTMA membrane. Therefore, it is confirmed that the DNA-LalanineC10 lipid complex film formed a more crystalline structure. However, under high humidity of $100 \%$, the X-ray refraction pattern of DNA-L-alanineC10 lipid film deformed and the refraction intensity decreased. It is assumed that water may cause a swelling effect of DNA to prevent the selfassembled structure.

\section{DNA-L-Glutamic Acid or L-Phenylalanine-derived Lipid Complex Film}

It was previously reported that physical properties of DNA-lipid complexes were greatly dependent on the kind of lipids in terms of alkyl chain length and number of alkyl chains. ${ }^{7}$ Since L-glutamic acid has two carboxylic acids which could be esterified by $\mathrm{C} 10$ alcohol to prepare LGluC10 lipid as mentioned in the synthetic method, a cast film was prepared from L-GluC10 to measure physical properties under $18 \%$ and $100 \%$ humidity. Results are shown in Figure 8.

It is seen in Figure 8 that the strain of DNA-L-GluC10 film was only $8 \mathrm{MPa}$ which is the same value as that of DNA-

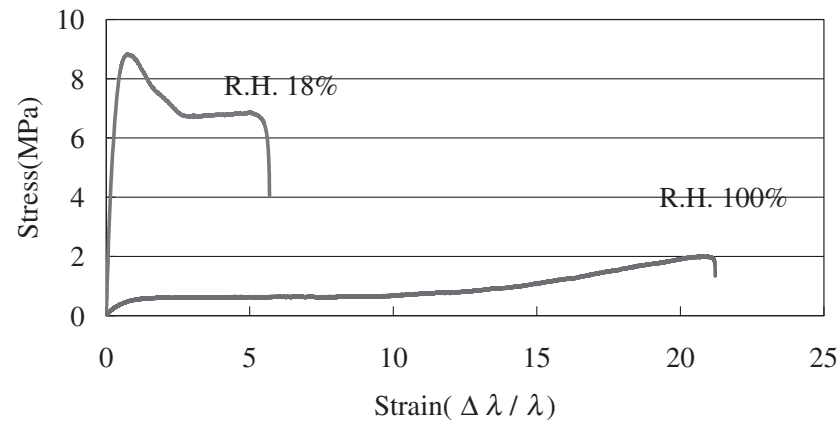

Figure 8. Physical properties of DNA-L-GluC10 lipid films under $18 \%$ and $100 \%$ relative humidity.

R.H. $0 \%$

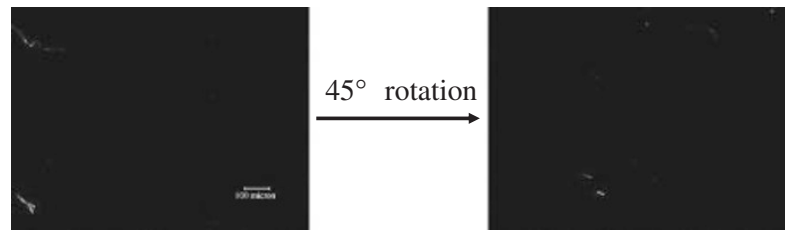

R.H. $100 \%$

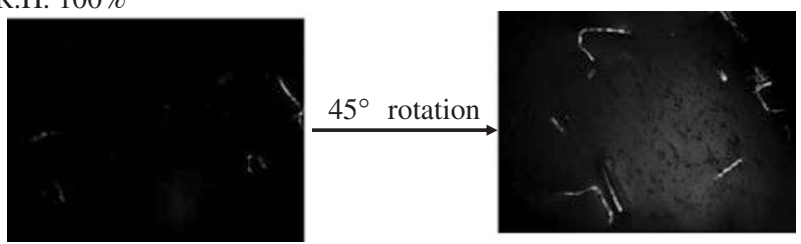

Figure 9. Cross-polarized microscopic pictures of DNA-L-GluC10 films under 0 and $100 \%$ relative humidity.

CTMA membrane, with much less elongation. Under $100 \%$ relative humidity the strain decreased to $2 \mathrm{MPa}$ with much elongation, owing to a plastisizing effect by water. These 


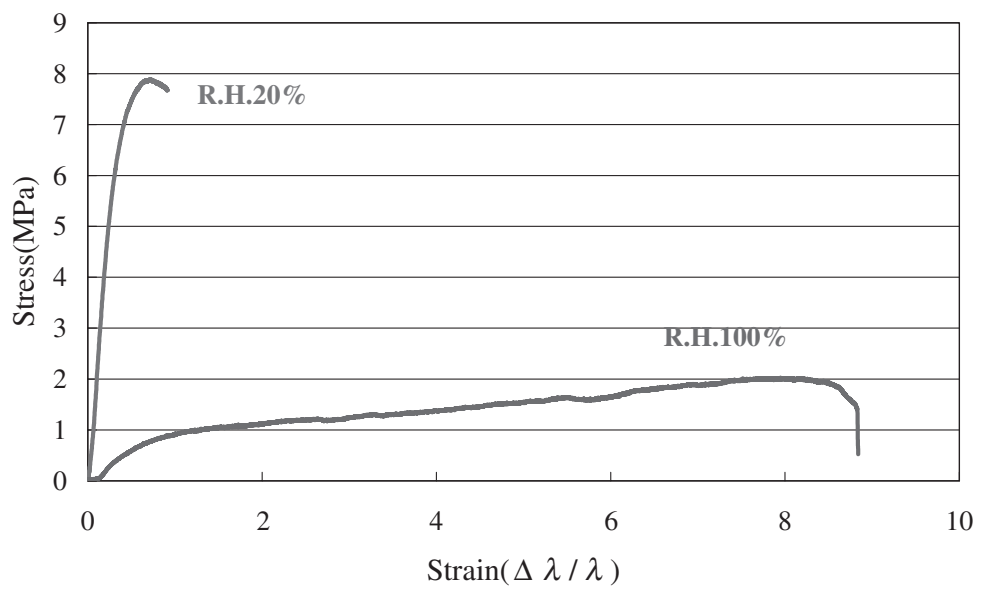

Figure 10. Physical properties of DNA-L-PhAIC10 lipid film under 20 and $100 \% \mathrm{RH}$.

results suggested a less self-assembled structure of the film of DNA-L-GluC10.

Cross-polarized mocroscopic pictures of the DNA-LGluC10 film are shown in Figure 9 which indicated no oriented structures under $0 \%$ relative humidity, while a little bright range was observed under $100 \%$ relative humidity, possible owing to a water-plasticizing effect. Therefore, the DNA-L-GluC10 lipid complex did not form a self-assembled structure which was clearly observed for the DNA-LalaninC10 complex. Two $\mathrm{C} 10$ alkyl groups surrounding DNA double helix may disturb the formation of the selfassembled structure.

Mechanical properties of DNA-L-Phenylalanine-derived lipid complex film are shown in Figure 10 which indicates that the mechanical strength at break is $8 \mathrm{MPa}$ under $20 \% \mathrm{RH}$, with an elongation of $3 \mathrm{~mm}$, while it decreases to $2 \mathrm{MPa}$ under $100 \% \mathrm{RH}$ with much longer elongation of $27 \mathrm{~mm}$, owing to a plastisizing effect by water. The mechanical strength of the DNA-L-PhAl C10 lipid complex film is almost similar as that of DNA-L-GluC10 lipid complex film, although the elongation of the DNA-L-GluC10 complex films is more than 3 times larger than that of DNA-L-PhAlC10 complex film, possibly owing to two C10 alkyl side groups of the DNA-L-GluC10 complex which would cause bulky spaces along DNA chains to enhance slipping during the elongation. Cross-polarized microscopic picture of the DNA-L-PhAlC10 complex film showed only a dark space after rotating $45^{\circ}$ and no oriented structure was observed. The bulky benzyl group of the DNA-L-PhAlC10 may disturb the orientation of the DNA-lipid complex, like the case of DNA-L-GluC10 complex film.

\section{CONCLUSION}

The as-cast DNA-L-alanineC10 complex film showed a selfassembled structure which was enhanced by water, while no self assembled structure was observed for the DNA-D- and racemic alanineC10 complex film. DNA-L-GluC10 lipid and -L-PhAl complex films did not form a self-assembled structure which was clearly observed for the DNA-L-alanineC10 complex. Two C10 alkyl groups of the GluC10 and benzyl group of the L-PhAlC10 lipids surrounding DNA double helix may disturb the formation of the self-assembled structure.

Received: April 15, 2007 Accepted: November 8, 2007 Published: January 29, 2008

\section{REFERENCES}

1. T. Tanaka and Y. Okahata, J. Am. Chem. Soc., 188, 10679 (1996)

2. N. Ogata, Kobunshi Ronbunshu, 61, 22 (2004).

3. M. Saito, K. Musha, T. Ikejima, S. Ozawa, Y. Yokoyama, and Y. Yokoyama, Kobunshi Ronbunshu, 6, 581 (2003).

4. A. Watanuki, H. Ikeda, J. Yoshida, and N. Ogata, Kobunshi Ronbunshu, 63, 419 (2006).

5. J. Yoshida, A. Watanuki, H. Takano, S. Kobayashi, H. Ikeda, and N. Ogata, Proc. SPIE, 6117, 61170L (2006).

6. N. Kobayashi, M. Hashimoto, and K. Kusabuka, Electrochemistry, 72, 440 (2004).

7. K. Yamaoka and N. Ogata, Kobunshi Ronbunshu, 61, 384 (2004).

8. C.-M. Wu, W. Lion, H.-L. Chen, T.-L. Lin, and U.-S. Jeng, Makromolecules, 37, 4974 (2004). 